Article

\title{
Early Warning to Insolvency in the Pension Fund: The French Case
}

\section{Noël Bonneuil ${ }^{1,2}$}

1 Institut national d'études démographiques, 133, bld Davout, 75980, Paris cedex 20, France;

E-Mail: bonneuil@ined.fr

2 École des hautes études en sciences sociales, 190 Avenue de France, F - 75013 Paris, France

Received: 9 November 2012; in revised form: 4 January 2013 / Accepted: 7 January 2013 /

Published: 18 January 2013

\begin{abstract}
The financial equilibrium of pension funds relies on the appropriate computation of retirement benefits, taking account of future payments and discount rates. Short-term errors in the commitment for retirement benefits, ill-suited investment in the stock market, or improper mixture with pay-as-you-go payments have long-term consequences and may lead the pension fund to insolvency. The differential equation governing the current assets shows the respective weights associated with the error on the interest rate, the error on the extra bonus, and the error made in forecasting mortality. These weights are estimated through simulations. A short follow-up is sufficient to estimate the three errors. A threshold for the extra interest rate to be earned on the financial market is given to counter-balance the extra bonus when mortality is forecast correctly.
\end{abstract}

Keywords: pension funding; retirement benefits; control differential equation; misestimation of mortality

\section{Introduction}

The sustainability of the pension fund relies on the strict observance of accounting equations. The objective of the fund normally is not to search for the best return at each time, but to find the appropriate balance between risk and gain so as to be able to pay the retirement benefits over the long period of liability [1]. However, managers of the fund are tempted to promise more than normally due from fair actuarial calculations, because the measures recommended against risk often contradict "the search for the optimal compromise between risk and return" [2] (p. 139). The French legislators have become 
aware of the danger, as it is reflected in the warrant of the 29 of November 2006 (Décret $n^{\circ} 2006-1499$ ) specifying the conditions of the reorganization of certain funds out of equilibrium.

For example, this warrant was invoked in the agreement of the 23rd of April 2007 establishing the contributions to the French pension fund for bailiffs (CARCO). An additional contribution of $4.1 \%$ was imposed to subscribers without benefits to be paid in the future, just for the sake of rescuing the fund. ${ }^{1}$ In fact, even from 2004 onwards, a contribution for solidarity of $2 \%$ was required for the same purpose of filling the gap between the so-called "special technical provision" and the so-called "mathematical technical provision", that is to say, to save the fund from insolvency. It was scheduled to cease after the gap was filled, but the additional contribution was instead increased to $4.1 \%$ in 2007. Moreover, this agreement contains an item whereby this additional contribution is allowed to be adjusted if the sum to be gained, namely 8,232,000 euros, is not attained.

A second example is the French pension fund for private hospitals (Caisse de retraite des établissements de soins privés (CRESP)). ${ }^{2}$ The fund declared a gap of 27.6 million euros in 2009 and of 41.4 million euros in 2010 between the theoretical and the recorded reserves. The ratio of the net present value of contributors to liabilities for beneficiaries (the "coverage ratio") was estimated at $86 \%$, then far from the value of $100 \%$ where it should be. The impossibility to pay benefits with the current parameters was explicitly acknowledged in $2003 .{ }^{3}$ The situation was explicitly assigned to the bad performance on the stock exchange. ${ }^{4}$

The French system works as a hybrid system. This is not exactly the same as a system with defined contributions [3]: at the moment of retirement, the capital acquired in the account of the employee is transformed into benefits. The employee acquires points, and the point has a value that can vary until the age of retirement where it is fixed by contract. The employee is promised a certain number of points. What is unknown is the value of the point at the moment of the settlement. In the normal working of a pension fund, each contributor assesses a certain amount of money to the fund on a regular basis, usually monthly. During his activity in the firm affiliated to the fund, the contributor is not allowed to withdraw his contribution. At retirement, the fund is committed to pay for a benefit by contract, which is due until death and can no longer be changed. The computation of the benefit is done from the capital invested by the contributor all along his period of contribution, augmented by the interest rate and by the mortality rate of other contributors dead in activity. It also takes into account the expected interest rate until the death of the contributor, and the expected mortality of other contributors (this is the principle of mutualization). By construction, sustainability of the fund is obtained through investment in predictable assets-which should involve little risk-through an acute forecasting of forthcoming mortality, and through respect of accounting rules. The difficulty comes from the fact that the fund must

\footnotetext{
1 "“...] une contribution additionnelle, ne donnant pas lieu à attribution d'unités de rente aux participants en application de l'article 4 de ce décret, fixée à 4,1\% de la rémunération brute des participants," source: http://www.legifrance.gouv.fr/.

${ }^{2}$ http://cresp-retraite.com/Rapport/rapport2010.htm $\sharp$ actuarielle.

3“[...] il a été constaté une insuffisance de réserves pour faire face aux engagements de retraite à long terme de nos cotisants."

4 “[...] une conjoncture boursière exceptionnellement à la baisse, des modifications réglementaires très défavorables ont conduit à réviser rétroactivement les droits ouverts aux cotisants avant 1999. Ainsi le coefficient de 1.1\% de la formule de calcul a été réduit sur la CRESP à un équivalent total de $1 \%$."
} 
balance present contributions with forthcoming pension benefits, and not, as in pay-as-you-go pensions, with benefits of the present pensioners.

Especially when the stock exchange is on the rise, the financier, the person in charge of yield profits from the capital of the fund, is tempted to bet on risky assets so as to maximize profit. But this is an error, because the financier, contrary to the pension fund, has no commitment to pay benefits on due date. The point is that the probability to reach the objective of benefit payments on due date is higher with long duration assets than with risky assets; it is that the logic of sure yields should supersede the logic of profit maximization, although the latter looks attractive. Similarly, longevity forecasts can under-estimate the actual improvement of survival, due to the rapid increase of hygiene and medicine, so that no annuity provider should determine the annuity rate solely on the basis of these forecasts. Finally, the manager, willing to attract more customers, should not be mistaken by current high rates of return on the stock market to promise more than the actuarial value of retirement benefits.

At the beginning of the fund, the scarcity of beneficiaries compared with contributors causes an important money flow into the fund.

- The temptation arises to increase the commitment for pensions beyond their due actuarial values in order to attract more contributors. This can be due to an inadequate culture of pay-as-yougo pensions, which favors short-sighted arbitrage, or to a hope of too optimistic returns from investments on the financial market through too risky assets.

- The fund fixes the value of the retirement benefits from the computation of the actuary done with a riskless long-term interest rate, but entrusts the management of the fund to a financier, who is not an insurer and invests in risky assets on the stock market. This can be due to a misunderstanding of the actuarial work and an inadequate trust in risk management [4,5].

- Errors can also come from too conservative a forecast of life expectancy. For example, as Cohen [6] pointed out, "the planners of the Social Security system in the United States during the 1930s failed to anticipate the remarkable improvement in the survival of older Americans." In July 2011, the French government lengthened the work duration of three months, because the national institute of statistical studies (INSEE) measured that life expectancy was increasing faster than expected. This is the third possible error that shall be addressed.

- The active population can also experience a drastic downsizing, which can have deleterious consequences if the fund, already out of equilibrium, is led to mutualize the contributions of different generations, that is to say, to use the funds affected to one generation to pay for another generation [7].

My overall objective is first to measure how a deviation is multiplied over time, because of the long run liability of the fund over the future, and second, to develop a warning aid through the differential equation giving the velocity (hence the rate) at which the current assets decrease. Ponds [8] was also concerned with solvency of the fund, but in a context of generational accounting related to defined-benefit schemes, which is less frequent than contribution-defined schemes, and he neither evaluated the rate of decline nor decomposed this rate among the types of error. 
In theory, only bonds should be used, but in the practical case that I present, managers have largely introduced risky assets into their capital. Solvency II norms provide a supplementary safety, but whatever the protections, fatal deviations are likely to remain. The argument of Solvency II amounts to have investments in stocks cost very high, but what will happen if firm bonds are no longer paid? Solvency II will not save everything, it will save only what is known, it is just a repair device of the deviations made so far. ${ }^{5}$

If the reorganization plan is taken, either by the managers of the fund who realize the coming insolvency or by a control authority, it usually relies on an increase of contributions of active contributors-sponsoring firm and active members of the plan-and on reduced retirement benefits of the contributors retiring after the date of the reorganization plan. For the two French pension funds I have mentioned, the duration of the re-organization plans were explicitly fixed to 20 years. The return to equilibrium is a function of controls and of the inherited liabilities at the date of the reorganization plan. One may wonder why does the government not control the pension funds, having in mind that such control would be beneficial? In France, pension funds have escaped the supervision of the control commission until now, but the government has recently indulged in extending the competence of this commission to organizations practicing insurance, through the mentioned warrant of 29 November 2006. However, pension funds in the industry are too numerous to be controlled in time. This explains why the person in charge of controlling the actors - contributors, financier, actuary, government - is the manager of the fund. He/she is then the person in charge of the solvency of the fund, unless an insurance company takes his/her place-in this case, the solvency of the fund is under control.

My contribution is firstly to enrich the general description done by [9] to the specific French case of pension funds and in focusing on the practical recent situation of French pension funds, secondly to suggest a warning device through the splitting up into its components of the slope with which deficits grow. This enables the minimal extra performance on the stock market to absorb the extra bonus and the possible error on mortality estimation. As soon as this warning signal is negative, measures are to be taken without waiting for the situation to worsen. My topic is again the practical case; it concerns neither participating contracts with with-profit bonuses, which do not concern France, nor adjustments of contributions or premiums, because contributions in France result from (long) negotiations between employees and employers and do not change easily, nor variable benefit accrual, which does not apply to French pension funds. From the analytical formula that I shall give, I shall evaluate the constructed early warning signal numerically, so as to present a practical indicator. I shall estimate the respective weights of the errors made on the interest rate, the extra bonus, and the mortality forecast.

\footnotetext{
${ }^{5}$ I must also add that the errors mentioned cannot be considered as frauds, and the process I am to describe has nothing to do with a Ponzi game: fund managers are not in fraud, they were not condemned, they acted (and still do) on good faith, the problem is that their normal competence has nothing to do with pension managing. They thus rely on two different advisers (financier and actuary), disconnected from each other, none of them accountable for his/her advice. This configuration (two disconnected advisers and a manager alien to professional pension managing) may be a source of the failure. Fund managers act on good faith. This is even why a law was promulgated in France. The very existence of the decree shows that this has been an error and not a fraud. What I show is that the fund had two opinions of good faith, each one incompetent on the other, and the problem where the failure appears is to unite them.
} 


\section{Model}

\subsection{Sustainable Working of Pension Funding}

For the sake of simplicity, I assume that assets associated with beneficiaries are invested at the same interest rate than those associated with still active contributors. The interest rate covers both a stochastic interest rate and stochastic stock returns, for I do not have to go in the detail of the portfolio.

At time $t$, cohort $\tau=t-a$ of age $a$ has contributed amount of:

$$
K_{g}(t-a, a)=\int_{0}^{a} e^{\int_{t-a+s}^{t} \hat{r}(u) d u} m_{g}(t-a, s) p_{g}(t-a, s) d s
$$

where $p_{g}(t-a, s)$ is the total number of contributors aged $s$ and born at time $t-a, m_{g}(t-a, s)$ is their instantaneous contributions per head into the fund at age $s$, usually taken as a fixed percent of wages, $\hat{r}(u)$ is the actual interest rate at time $u$ of assets bought for still active people. I shall use the index $g$ to specify that the first argument of the associated function corresponds to the number of the cohort.

The capital gathered by cohort $t-a$ at age $\omega$ of retirement (assumed constant in time) is then returned to beneficiaries through retirement benefits $c_{g}(t-a, s)$ for one euro at age $s$ until maximal age $\omega_{\dagger}$ of life, so that the capital left by cohort $t-a$ at age $a=\omega, \cdots, \omega_{\dagger}$ is:

$$
K_{g}(t-a, \omega)\left(e^{\int_{t-a+\omega}^{t} r(u) d u}-\int_{\omega}^{a} c_{g}(t-a, b) e^{\int_{t-a+b}^{t} r(u) d u} l_{g}(t-a, b) d b\right)
$$

which is the continuous version of classical actuarial calculus and where $l_{g}(t-a, b)$ is the expected cohort probability of surviving from age $\omega$ to age $b \in\left[\omega, \omega_{\dagger}\right]$ :

$$
l_{g}(t-a, b)=e^{-\int_{\omega}^{b} \mu(t-a+u, u) d u}
$$

with $\mu(t, x)$ the usual continuous-time force of mortality at time $t$ and age $x$. I denote $\hat{l}_{g}(t-a, b)$ as the actual survival probability and $l_{g}(t-a, b)$ as the expected one.

The accounting balance of the fund giving the value $c_{g}(\tau, b)$ for generation $\tau$ of the pension for one euro for cohort $\tau$ and age $b$ is obtained when the entire capital gathered by cohort $\tau$ has been entirely paid back to beneficiaries of cohort $\tau$, after the percentages taken by the fund (at entry, on the interest rate, or at sale) which do not appear in the equations for the sake of clarity:

$$
\forall \tau, e^{\int_{\tau+\omega}^{\tau+\omega_{\dagger}} r(u) d u}=\int_{\omega}^{\omega_{\dagger}} c_{g}(\tau, b) e^{\int_{\tau+b}^{\tau+\omega_{\dagger}} r(u) d u} l_{g}(\tau, b) d b
$$

For $c_{g}(\tau, b)=c_{g}(\tau)$ constant with age,

$$
c_{g}(\tau)=\frac{e^{\int_{\tau+\omega}^{\tau+\omega_{\dagger}} r(u) d u}}{\int_{\omega}^{\omega_{\dagger}} e^{\int_{\tau+b}^{\tau+\omega_{\dagger}} r(u) d u} l_{g}(\tau, b) d b},
$$

which is the continuous-time version of the classical actuarial calculus of retirement benefits.

The annual discrete-time version of Equation (5) is:

$$
c_{g}(\tau)=\frac{\prod_{u=\tau+\omega}^{\tau+\omega_{\dagger}-1} r(u)}{\sum_{b=\omega}^{\omega_{\dagger}-1} \Pi_{u=\tau+b}^{\tau+\omega_{\dagger}-1}(1+r(u)) \frac{1}{2}\left(l_{g}(\tau, b)+l_{g}(\tau, b+1)\right)},
$$

where $r(u)$ denotes the mean interest rate for year $u$. 


\subsection{Deviation from Actuarial Equilibrium}

The percentages received by the fund at sale of the contract are incentives for promising more attractive pensions, especially if the fund benefits from a favorable ratio of active/beneficiaries and if the financial market lets people hope for high returns. In certain funds, the contribution is used to buy points. The price of a point can vary over time. The total number of points acquired at retirement time is changed into pension benefits. The error consists in allowing bonus points to contributors, hence a commitment for higher retirement benefits for one euro. In other funds without points, the mechanism of allowing bonus is similar. For one euro at the beginning of the contract (at retirement), a constant $c_{g}(\tau)(1+\delta)$ is promised instead of the constant $c_{g}(\tau)$ at equilibrium of Equation (5).

Because the fund is bound by contract to pay for the beneficiaries until their deaths, through Equation (4) or Equation (5), any temporary deviation from this accounting balance engages the fund for decades and for its corresponding deficit. The error then runs from the beginning to the point of the reorganization plan (as observed in practice, benefits and points allocations change only slowly because they are decided after negotiations between employees and employers). The entire capital gathered by cohort $\tau$ is entirely paid back to beneficiaries of cohort $\tau$. Although the environment is uncertain, the pension fund makes sure "fair" promises to the cohort at retirement. This assumption explains why, even small, an extra bonus leads to insolvency.

The fund can be in permanent sustainable regime when the deviation occurs. However, practically, bonus points are allowed shortly after the creation of the fund, at $t_{0}$, so, because of the disproportionate length of liability compared with the period of working of the fund, it is more realistic to consider that the deviation is made from the very beginning of the fund.

Depending on the contract, the capital paid to the fund by an individual who dies before retiring is either paid back to the spouse, paid as a lump sum, or, by far the most frequent case, it remains in the fund and is used in the mutualization system to increase retirement benefits paid to survivors. I focus on this latter case to study the consequences of an extra bonus in the account of the fund. Moreover, mortality in most developed countries where pension funding can exist is sufficiently low before usual age of retirement to discard the first option.

Three deviations can accumulate: the fund is committed by contract to pay more than $c_{g}$, say $(1+\delta) c_{g}$ in order to capture subscribers; the interest rate has been forecast as $r(t)$ but its realization is $\hat{r}(t)$; the survival has been forecast as $l_{g}$ but its realization is $\hat{l}_{g}$. The payment $c_{g}$ is stipulated in a contract, then it cannot be adjusted dynamically. To change $c_{g}$, the trade unions and the management of the firm must agree on a new contract, which is hard to obtain. This happens when the plan is reorganized.

The solvency of the fund depends on three accounts, corresponding to three groups of people:

- The current assets for one euro and for a beneficiary of generation $t-a<t-\omega_{\dagger}$ (then dead at $t$ ) is:

$$
\lambda_{D}(t-a):=e^{\int_{t-a+\omega}^{t-a+\omega_{\dagger}} \hat{r}(u) d u}-(1+\delta) c_{g}(t-a) \int_{\omega}^{\omega_{\dagger}} e^{\int_{t-a+b}^{t-a+\omega_{\dagger}} \hat{r}(u) d u} \hat{l}_{g}(t-a, b) d b
$$

which is null if $\delta=0, \hat{l}_{g}=l_{g}$, and $\hat{r}=r$. If $\delta>0$, it can also be non-negative if also $\hat{r}>r$, which is exactly the bet made by financiers in a context of rising stock exchange. 
Late cohorts $t_{0}-\omega_{\dagger}, \cdots, t-\omega_{\dagger}$, where $t_{0}$ is the starting date of the fund, have left a deficit at time $t$ of:

$$
x_{D}(t):=\int_{t_{0}-\omega_{\dagger}}^{t-\omega_{\dagger}} K_{g}(\tau, \omega) \lambda_{D}(\tau) d \tau
$$

- The current assets remaining for one euro and for a beneficiary of age $a \in\left[\omega, \omega_{\dagger}\right]$ at time $t$ is:

$$
\lambda_{P}(t-a, a):=e^{\int_{t-a+\omega}^{t} \hat{r}(u) d u}-(1+\delta) c_{g}(t-a) \int_{\omega}^{a} e^{\int_{t-a+b}^{t} \hat{r}(u) d u} \hat{l}_{g}(t-a, b) d b
$$

At time $t$, present beneficiaries have a capital of:

$$
x_{P}(t):=\int_{\omega}^{\omega_{\dagger}} K_{g}(t-a, \omega) \lambda_{P}(t-a, a) d a=\int_{t-\omega_{\dagger}}^{t-\omega} K_{g}(\tau, \omega) \lambda_{P}(\tau, t-\tau) d \tau
$$

- The capital brought by contributors in activity is:

$$
x_{A}(t):=\int_{0}^{\omega} K_{g}(t-a, a) d a
$$

Expected liabilities for one euro and for these future beneficiaries are $(1+\delta) c_{g}(t-a)$.

The current assets of the fund are:

$$
x(t):=x_{D}(t)+x_{P}(t)+x_{A}(t) .
$$

Time elapsed since the beginning of the fund appears only in $x_{D}(t)$ : for $\delta>0$, the accumulation of losses is linear in time, after the initial phase of the arrival of beneficiaries to be paid by the fund, causing the coefficient of linearity to decrease to its permanent regime, depending only on contributions, interest rate, and survival. The term independent of duration $x_{P}(t)+x_{A}(t)$ starts from 0 at the beginning of the fund to increase to its level of permanent regime.

The dynamic of debt left by the dead is:

$$
\frac{\partial x_{D}(t)}{\partial t}=K_{g}\left(t-\omega_{\dagger}, \omega\right) e^{\int_{t-\omega_{\dagger}+\omega}^{t} \hat{r}(u) d u}\left(1-(1+\delta) \frac{c_{g}\left(t-\omega_{\dagger}\right)}{\hat{c}_{g}\left(t-\omega_{\dagger}\right)}\right)
$$

with

$$
\hat{c}_{g}(\tau)=\frac{e^{\int_{\tau+\omega}^{\tau+\omega_{\dagger}} \hat{r}(u) d u}}{\int_{\omega}^{\omega_{\dagger}} e^{\int_{\tau+b}^{\tau+\omega_{\dagger}} \hat{r}(u) d u} \hat{l}_{g}(\tau, b) d b} .
$$

From Equation (10), second expression, the dynamic of the capital related to the pensioners is:

$$
\begin{gathered}
\frac{\partial x_{P}(t)}{\partial t}=\int_{t-\omega_{\dagger}}^{t-\omega} K_{g}(\tau, \omega)\left(\hat{r}(t) \lambda_{P}(\tau, t-\tau)-c_{g}(\tau)(1+\delta) \hat{l}_{g}(\tau, t-\tau)\right) d \tau \\
+K_{g}(\tau-\omega, \omega) \\
\quad-K_{g}\left(t-\omega_{\dagger}, \omega\right) e^{\int_{t-\omega_{\dagger}+\omega}^{t} \hat{r}(u) d u}\left(1-(1+\delta) \frac{c_{g}\left(t-\omega_{\dagger}\right)}{\hat{c}_{g}\left(t-\omega_{\dagger}\right)}\right)
\end{gathered}
$$

Summing Equation (13) and (15) yields:

$$
\frac{\partial x_{D}(t)}{\partial t}+\frac{\partial x_{P}(t)}{\partial t}=\hat{r}(t) x_{P}(t)+K_{g}(t-\omega, \omega)-(1+\delta) \int_{t-\omega_{\dagger}}^{t-\omega} K_{g}(\tau, \omega) c_{g}(\tau) \hat{l}_{g}(\tau, t-\tau) d \tau
$$

where the first term $\hat{r}(t) x_{P}(t)$ is the interest over the debt, the second term $K_{g}(t-\omega, \omega)$ is the capital entering the current assets for the old and the dead, and the third term multiplied by $d t$ represents the payment of benefits between $t$ and $t+d t$. 
Equation (16) yields the growth rate of the sum of the deficit left by late cohorts and the current assets for pensioners. When this growth rate is negative, this is an early warning signal sent to the manager of the fund.

I suggest now to examine simplified versions of this key Equation (16), in order to assess the relative importance of the error on the interest rate, the error on payment of benefits, and the error on mortality forecasting.

\subsection{The Relative Importance of Each Error}

At $c_{g}$ and $K_{g}$ constant,

$$
\frac{\partial x_{D}(t)}{\partial t}+\frac{\partial x_{P}(t)}{\partial t}=\hat{r}(t) x_{P}(t)+\left(1-c_{g}(1+\delta) \hat{e}_{\omega_{\dagger} / \omega}\right) K_{g}
$$

where

$$
\hat{e}_{\omega_{\dagger} / \omega}(t)=\int_{t-\omega_{\dagger}}^{t-\omega} \hat{l}_{g}(\tau, t-\tau) d \tau=\int_{\omega}^{\omega_{\dagger}} e^{-\int_{\omega}^{a} \hat{\mu}(t-a+u, u) d u} d a
$$

resembles the expression of the actual life expectancy at age $\omega$, except that survival functions are taken by generation, and generations vary. ${ }^{6}$

For $\delta=0, \hat{r}(t)=r(t), \hat{\mu}(t, a)=\mu(t, a)$, actuarial equations imply:

$$
\left.r(t) x_{P}(t)\right|_{\hat{r}=r, \delta=0, \hat{\mu}=\mu}+\left(1-c_{g} e_{\omega_{\dagger} / \omega}(t)\right) K_{g}=0
$$

with

$$
e_{\omega_{\dagger} / \omega}(t)=\int_{t-\omega_{\dagger}}^{t-\omega} l_{g}(\tau, t-\tau) d \tau=\int_{\omega}^{\omega_{\dagger}} e^{-\int_{\omega}^{a} \mu(t-a+u, u) d u} d a,
$$

which is a sort of life expectancy at age $\omega$, but again with both generation and time varying.

Define $\Delta r(t)$ as:

$$
\Delta r(t)=\hat{r}(t)-r(t)
$$

and $\Delta \mu(t, u)$ as:

$$
\Delta \mu(t, u)=\hat{\mu}(t, u)-\mu(t, u),
$$

where $\hat{\mu}$ is the actual mortality rate. In the past, the variations of mortality have concerned all ages. There has not been such a thing as a mortality intensity changing at a given age and unchanged at other ages. This allows me to assume that the error on mortality forecast is approximately the same at all ages over $\omega$ :

$$
\Delta \mu(t, u)=\Delta \mu(t)
$$

Subsequently,

$$
\hat{l}_{g}(t, b)=l_{g}(t, b) e^{-\Delta \mu(t)(b-\omega)}
$$

From the French life tables ${ }^{7} 2004-6,2005-7,2006-8,2007-9$, and 2008-10, I estimated $\mu(t+1, a)-$ $\mu(t, a)$ at each age and successively at 2005, 2006, 2007, 2008, and 2009 from $a=65$ to $a=95$ years

\footnotetext{
${ }^{6}$ The generation life expectancy for generation $g$ is $\int_{\omega}^{\omega_{\dagger}} e^{-\int_{\omega}^{a} \hat{\mu}(g+u, u) d u} d ? a$ and the period life expectancy at time $t$ is $\int_{\omega}^{\omega_{\dagger}} e^{-\int_{\omega}^{a} \hat{\mu}(t, u) d u} d a$.

${ }^{7}$ Source: France métropolitaine, territoire au 31 décembre 2010, Insee, statistiques de l'état civil et estimations de population.
} 
of age, as a proxy for $\Delta \mu(t, a)$, which turns out to be approximately independent of age $a(\Delta \mu(t, a) \approx$ $\Delta \mu(t))$ : the error $\Delta \mu(t)$ made in forecasting should be of the same order of magnitude than the yearly actual variation, notably if mortality is forecast at year $t+1$ as the value taken at year $t$. On these data, I found that $(\mu(t+1, b)-\mu(t, b))(b-\omega)$, for $\omega=65$ and $b=65, \cdots, 95$, is equal to -0.0149 ( $\mathrm{SD}=0.0151)$ on average for men and to -0.0163 ( $\mathrm{SD}=0.013)$ for women. These low values allow the assumption $|\Delta \mu(b-\omega)| \ll 1$, for $b=65, \cdots, 95$, which leads to the first-order approximation of the "life expectancy" at $t$ :

$$
\begin{aligned}
\hat{e}_{\omega_{\dagger} / \omega}(t) & =\int_{\omega}^{\omega_{\dagger}} \hat{l}_{g}(t-b, b) d b \\
& =\int_{\omega}^{\omega_{\dagger}} l_{g}(t-b, b) e^{-\Delta \mu(t)(b-\omega)} d b \\
& \approx e_{\omega_{\dagger} / \omega}(t)-\Delta \mu(t) \int_{\omega}^{\omega_{\dagger}}(b-\omega) l_{g}(t-b, b) d b \\
& =e_{\omega_{\dagger} / \omega}(t)-\Delta \mu(t)\left(\left(\omega_{\dagger}-\omega\right) e_{\omega_{\dagger} / \omega}(t)-\int_{\omega}^{\omega_{\dagger}} e_{b / \omega_{\dagger}}(t) d b\right) \\
& =e_{\omega_{\dagger} / \omega}(t)-\Delta \mu(t) \int_{\omega}^{\omega_{\dagger}} e_{\omega_{\dagger} / b}(t) d b
\end{aligned}
$$

For $\Delta r(t) \ll r(t)$ and $\delta \ll c_{g}$, using Equation (19) and taking $\frac{\partial x_{P}(t)}{\partial r}$ and $x_{P}(t)$ at $\hat{r}=r$ and $\delta=0$, the variation of the amount of current assets is:

$$
\left\{\begin{aligned}
& \frac{\partial x_{D}(t)}{\partial t}+\frac{\partial x_{P}(t)}{\partial t} \approx\left(r(t) \frac{\partial x_{P}(t)}{\partial r}+x_{P}(t)\right) \Delta r(t) \\
&+\left(r(t) \frac{\partial x_{P}(t)}{\partial \delta}-c_{g} K_{g} e_{\omega_{\dagger} / \omega}(t)\right) \delta \\
& \quad+\left(r(t) \frac{\partial x_{P}(t)}{\partial \mu}+K_{g} c_{g} \int_{\omega}^{\omega_{\dagger}} e_{\omega_{\dagger} / b}(t) d b\right) \Delta \mu(t) \\
& \quad K_{g} \int_{t-\omega_{\dagger}}^{t-\omega}\left(y(\tau+\omega, t) e^{\int_{\tau+\omega}^{t} r(u) d u}\right. \\
&\left.\quad-c_{g} \int_{\omega}^{t-\tau} y(\tau+b, t) e^{\int_{\tau+b}^{t} r(u) d u} l_{g}(\tau, b) d b\right) d \tau \\
& \frac{\partial x_{P}(t)}{\partial r}= \Delta r(t) y(\tau, t), \quad \forall \tau \leq t, \quad y(t, t)=0 \\
& \frac{\partial y(\tau, t)}{\partial t}=-K_{g} c_{g} \int_{t-\omega_{\dagger}}^{t-\omega}\left(\int_{\omega}^{t-\tau} e^{\int_{\tau+b}^{t} r(u) d u} l_{g}(\tau, b) d b\right) d \tau \\
& \frac{\partial x_{P}(t)}{\partial \delta}= K_{g} c_{g} \int_{t-\omega_{\dagger}}^{t-\omega}\left(\int_{\omega}^{t-\tau} e^{\int_{\tau+b}^{t} r(u) d u}(b-\omega) l_{g}(\tau, b) d b\right) d \tau \\
& \frac{\partial x_{P}(t)}{\partial \mu}=
\end{aligned}\right.
$$

which is a differential system with state variables $x_{D}(t), x_{P}(t)$, and $y(\tau+\omega, t)$ (defined in the third equation of System (26)), $\tau=t_{0}, \cdots, t$ and linear controls $\Delta r(t), \delta$, and $\Delta \mu(t)$, except that $\delta$ is fixed by contract and cannot be changed dynamically and except that $\Delta r(t)$ and $\Delta \mu(t)$ are exogenous.

At the first-order, the term $\frac{\partial x_{P}(t)}{\partial r} \Delta r(t)$ vanishes, and Equation (26) boils down to:

$$
\begin{aligned}
\frac{1}{K_{g}}\left(\frac{\partial x_{D}(t)}{\partial t}+\frac{\partial x_{P}(t)}{\partial t}\right) \approx & \left(\int_{t-\omega_{\dagger}}^{t-\omega}\left(e^{\int_{\tau+\omega}^{t} r(u) d u}-c_{g} \int_{\omega}^{t-\tau} e^{\int_{\tau+b}^{t} r(u) d u} l_{g}(\tau, b) d b\right) d \tau\right) \Delta r(t) \\
& -c_{g}\left(r(t) \int_{t-\omega_{\dagger}}^{t-\omega} \int_{\omega}^{t-\tau} e^{\int_{\tau+b}^{t} r(u) d u} l_{g}(\tau, b) d b d \tau+e_{\omega_{\dagger} / \omega}(t)\right) \delta \\
& +c_{g}\left(r(t) \int_{t-\omega_{\dagger}}^{t-\omega} \int_{\omega}^{t-\tau} e^{\int_{\tau+b}^{t} r(u) d u} l_{g}(\tau, b)(b-\omega) d b d \tau\right. \\
& \left.+\int_{\omega}^{\omega_{\dagger}} e_{\omega_{\dagger} / b}(t) d b\right) \Delta \mu(t)
\end{aligned}
$$

where, consistently, the extra bonus $(\delta>0)$ adds a negative term, as do an under-estimation of mortality $(\Delta \mu(t)<0)$ and a bad performance $\Delta r(t)<0$ on stocks (the coefficients of $\Delta r$ and of $\delta \mu$ are positive by construction). Equation (27) is an original result: it completes the display of information on the economic health of the fund. It adds the detail of the components of the velocity $\frac{\partial x_{D}(t)}{\partial t}+\frac{\partial x_{P}(t)}{\partial t}$ to the state $x_{D}(t)+x_{P}(t)$ of the current assets; it states how the stock exchange should extra-perform the actuarial rate at each date to rescue the situation endangered by the allowance of the extra bonus or by an unexpected improvement of mortality at old ages. It tells which component is responsible for the 
variations of $x_{D}(t)+x_{P}(t)$; it allows the manager to assess his/her chances to rescue the situation in the case of a declining amount of current assets.

The variable $\xi(t):=\frac{1}{K_{g}}\left(\frac{\partial x_{D}(t)}{\partial t}+\frac{\partial x_{P}(t)}{\partial t}\right)$ behaves as an early warning signal that the pension fund has made errors that compromise its solvency in the future. The simple fact that $x_{D}+x_{P}$ is decreasing is already enough to warn the manager that something is going wrong; $\xi(t)$ specifies the importance of each error. As we shall see below, we can even estimate the values of each error $\Delta r(t), \delta$, and $\Delta \mu$ from the follow-up of $\xi(t)$ over four time units, a piece of information that the sole knowledge of $x_{D}(t)+x_{P}(t)$ does not allow.

In the noteworthy case of a perfect forecasting of mortality, it is not enough that $\Delta r(t)>0$, but, from Equation (27), the manager should have $\frac{\partial x_{D}(t)}{\partial t}+\frac{\partial x_{P}(t)}{\partial t} \geq 0$, or:

$$
\Delta r(t) \geq \frac{e_{\omega_{\dagger} / \omega}(t)+r(t) \int_{t-\omega_{\dagger}}^{t-\omega} \int_{\omega}^{t-\tau} e^{\int_{\tau+b}^{t} r(u) d u} l_{g}(\tau, b) d b d \tau}{\int_{t-\omega_{\dagger}}^{t-\omega}\left(e^{\int_{\tau+\omega}^{t} r(u) d u}-c_{g} \int_{\omega}^{t-\tau} e^{\int_{\tau+b}^{t} r(u) d u} l_{g}(\tau, b) d b\right) d \tau} c_{g} \delta
$$

In case of too high retirement benefits $c_{g}$, the denominator is negative and, as the numerator is positive, $\Delta r(t)$ should be positive. Equation (28) gives a clear rule of how the return on the stock exchange must extra-perform to counterbalance the extra bonus. Actuaries advise $\delta=0, \Delta r(t)=0$, and $\Delta \mu(t)=0$, but financiers allowing the extra bonus $\delta$ bet that $\Delta r(t)$ will be high enough to ensure that $\frac{\partial x_{D}(t)}{\partial t}+\frac{\partial x_{P}(t)}{\partial t}>0$, having no knowledge really on $\frac{\partial x_{A}(t)}{\partial t}$. Extra bonuses are allowed for commercial purposes, without measuring the long-term consequences on the solvency of the fund, trusting the stock exchange to fill the gap. Equation (27) or Equation (28) allow the computation of the necessary extra asset return $\Delta r(t)$ : if it is unrealistic, the manager had better re-organize the fund without waiting for $x(t)$ to be low enough. The problem is that $\Delta r(t)$ can be less than the threshold given by Equation (27) or Equation (28), even negative for a long period of time, as what happened during the financial crisis of 2008-2009. Then Equation (27) clearly indicates the weight of each error $\Delta r(t), \delta$, and $\Delta \mu(t)$ in the short term. An estimate of the time to insolvency when the current assets are $x(t)$ at time $t$ is $x(t) /\left(A+\frac{\partial x_{A}(t)}{\partial t}\right)$, where $A$ is the right hand side of Equation (27).

\subsection{The Early Warning Signal in Practice}

Discrete-time Expression One annual discrete-time approximation of Equation (27) is:

$$
\begin{aligned}
\frac{\partial x_{D}(t)}{\partial t}+\frac{\partial x_{P}(t)}{\partial t} \approx & K_{g}\left(\left(\sum _ { \tau = t - \omega _ { \dagger } } ^ { t - \omega } \left(\prod_{u=\tau+\omega}^{t-1} r(u)\right.\right.\right. \\
& \left.\left.-c_{g} \sum_{b=\omega}^{t-\tau-1} \prod_{u=\tau+b}^{t-1} r(u) \frac{1}{2}\left(l_{g}(\tau, b)+l_{g}(\tau, b+1)\right)\right)\right) \Delta r(t) \\
& -\left(e_{\omega_{\dagger} / \omega}(t)+r(t) \sum_{\tau=t-\omega_{\dagger}}^{t-\omega-1} \sum_{b=\omega}^{t-\tau-1}\left(\prod_{u=\tau+b}^{t-1} r(u)\right.\right. \\
& \left.\times \frac{1}{2}\left(l_{g}(\tau, b)+l_{g}(\tau, b+1)\right)\right) c_{g} \delta \\
+ & \left(\sum_{\tau=t-\omega_{\dagger}}^{t-\omega-1} \sum_{b=\omega}^{t-\tau-1} \prod_{u=\tau+b}^{t-1} r(u)(b-\omega) \frac{1}{2}\left(l_{g}(\tau, b)+l_{g}(\tau, b+1)\right)\right. \\
& \left.\left.+\sum_{b=\omega}^{\omega_{\dagger}} e_{\omega_{\dagger} / b}(t)\right) c_{g} \Delta \mu\right)
\end{aligned}
$$

This formula can be used for practical computation.

Numerical values I simulated 200 time series of interest rates $r(t)$ and life tables $\mu(t, u), u=0,99$, $t=1, \cdots, 50$. The life tables were generated from Ledermann model life tables [10]. For each time 
series, I simulated $\delta$ drawn at random in $[0,0.3], \Delta e_{0}:=\hat{e}_{0}-e_{0}$ in $[-6,6]$ years, the error made on the forecast of the life expectancy at birth $e_{0}$, itself drawn at random between 75 and 90 years. $\Delta \mu$, the $\hat{l}_{g}$, and the $l_{g}$ are deduced from $\Delta e_{0}$. The standard deviation $\sigma_{r}$ of the interest rate was drawn at random from $[0 \%, 5 \%]$, so that $\hat{r}(t)$ was taken from a Gaussian distribution $\mathcal{N}\left(\bar{r}, \sigma_{r}\right)$, with $\bar{r}$ drawn at random from $[-3 \%, 3 \%]$. The error on the interest rate $\Delta r(t)=\Delta r$ was drawn at random in $[-3 \%, 3 \%]$,

Time to Insolvency On the 128 simulated time-series for which $x_{D}(t)+x_{P}(t)$ decreased until becoming negative, I found a significant positive correlation, as expected, between $\xi(t)$ and the first time $t_{\min }$ when $x_{D}(t)+x_{P}(t)<0$ for the first time. The regression over the 128 trials of the mean value $\bar{\xi}$ of $\xi(t)$ over time on $t_{\min }$ yields:

$$
E(\xi)=-\underset{(0.0378)}{0.5753^{*}}+\underset{(0.0003)}{0.0020^{*}} t_{\min }
$$

where the star indicates statistical significance at the $5 \%$ level and standard deviations are put in parentheses below their coefficients. Equation (30) shows that the more negative $E(\xi)$, the sooner the moment when $x_{D}+x_{P}<0$. Moreover, for the 128 simulated time series for which $x_{D}+x_{P}<0$ before the time horizon of the simulation, on average, $E(\xi)=-\underset{(0.387)}{0.432}$, which shows that $E(\xi)$ is significantly negative when the pension fund is doomed to insolvency (net the effect of the capital brought by contributors in activity).

Numerical Evaluation of the Early Warning Signal From these 128 simulated time series, I estimated the coefficients associated with each of the three errors in Equation (27) (or Equation (29)) as:

$$
\begin{array}{rc}
\text { coefficient of } \Delta r(t): & -\underset{(11.12)}{36.97^{*}}-\underset{(48.21)}{22.66} \bar{r}+\underset{(0.13)}{0.59^{*}} \bar{e}_{0}(t) \\
\text { coefficient of } \delta: & -\underset{(0.64)}{0.39}-\underset{(2.78)}{16.03^{*}} \bar{r}-\underset{(0.008)}{0.008} \bar{e}_{0}(t) \\
\text { coefficient of } \Delta \mu: & \underset{(12.75)}{40.93^{*}}+\underset{(55.24)}{356.35 *} \bar{r}-\underset{(0.15)}{0.21} \bar{e}_{0}(t)
\end{array}
$$

The three respective coefficients of $\Delta r(t), \delta$, and $\Delta \mu(t)$ can be computed by the fund manager, because they are built from forecast interest rates and mortality, not from the actual values of these variables. The manager can also compute $\xi(t)$ directly from the knowledge of $x_{D}(t)+x_{P}(t)$ followed up over four successive time units. Then, by solving a linear system of three equations identifying $\left(x_{D}(t+1)+x_{P}(t+\right.$ $1))-\left(x_{D}(t)+x_{P}(t)\right)$ to a linear combination of $\Delta r(t), \delta$, and $\Delta \mu(t)$, for $t$ taking four successive values and the coefficients estimated as in Equation (31), the manager can estimate the three errors $\Delta r(t), \delta$, and $\Delta \mu(t)$. The manager reiterates the procedure at each time unit to estimate how the errors vary in time. I tested it numerically and the estimation works very well to find again the input values of $\Delta r(t)$, $\delta$, and $\Delta \mu(t)$.

Moreover, on the 128 simulated time series leading to $x_{D}(t)+x_{P}(t)<0$, I find the weights of each error appearing in Equation (27) (as means of the coefficients expressed in Equation (31)), with standard deviations in parentheses below mean values, as:

$$
E(\xi)(t)=\underset{(4.13)}{12.84} \Delta r(t)-\underset{(0.29)}{1.19} \delta+\underset{(6.32)}{25.71} \Delta \mu(t)
$$


which shows the prior importance of a good prediction of mortality. Mortality intervenes in the computation of retirement benefits for one euro and in deficits. The manager should under-estimate mortality from the start, but technological progress is so unpredictable that mortality can decline faster than expected. The second message of Equation (32) is that the weight of $\Delta r$ is high enough that, definitely, the manager should not invest in risky assets where errors are more likely to occur than with riskless assets. Finally, the coefficient of $\delta$, the error made on retirement benefits for one euro, is the lowest one. However, there is no biological intuition as for $\Delta \mu$ or economic guess as for $\Delta r$ to prevent the manager from offering a high $\delta$. Even with a low weight in Equation (32), a high $\delta$ can prove as deleterious for the pension solvency as the other errors weighted by higher coefficients.

\section{Conclusions}

I have examined the consequences of deviations on the sustainability of the fund. I have highlighted how errors, either on investment or in payment of benefits, are multiplied by the liability structure and its long-term kinematics. Developing on actuarial equations, I showed notably that even a small extra bonus $(\delta)$ of retirement benefits is enough to carry the fund away into deficits, which grow linearly with time. I expressed the current assets analytically, and wrote the differential equation (27) expressing the velocity with which this amount of current assets varies. I deduced the minimal excess return on stocks (Equation (28)) necessary to counter-balance the extra bonus and the error on mortality forecasting if any. I deduced an estimate at each time $t$ of the time to insolvency.

Population size and age structure in a pension fund should play no role, because each contributed euro corresponds to a benefit. However, the necessity to pay for the deficits left by dead cohorts leads the fund to mutualize the contributions, giving a role to the liability structure and hence to population stocks. However, the mutualization does not rescue the fund in the mean term. Equation (27) helps knowing if the entry of new capital $\frac{\partial x_{A}(t)}{\partial t}$ is enough to counter-balance deficits and push $x^{\prime}(t)$ to positive values, the manager having the responsibility to lower the benefit to attain $\delta=0$ with the new entrants. Otherwise, an insufficient $\Delta r(t)$ runs off the fund to insolvency, and a reorganization plan (consisting of fixing a $\delta<0$ temporarily) is mandatory, for fear of insolvency.

The policy recommendation is that the French State asks the commission for auditing; the very basis of the Marini [11] report and of the warrant of 29 November 2006 is to control liabilities and assets together. My contribution is to recapitulate the process leading a pension fund to insolvency, to write down Equation (27) that clarifies the weight of each component in the decline of the current assets, and finally to pose the constraint that the early warning signal defined by Equation (27) must be non-negative $(\xi(t) \geq 0)$. This warning signal helps assess the chances to rescue the fund through the stock exchange, and in case that this issue is unlikely, to accelerate the decision to re-organize the plan before the amount of current assets has fallen down to low values.

\section{References}

1. Bowers, N.L.; Hickman, J.C.; Nesbitt, C.J. Introduction to the dynamics of pension funding. Transactions of the Society of Actuaries 1976, 28, 177-203. 
2. El Mekkaoui-de Freitas, Najat. Réglement et choix de portefeuille des fonds de pension. In Fonds de Pension; Bismut, C., El Mekkaoui-de Freitas, N., Eds.; Economica: Paris, France, 2000; pp.129-143.

3. Blake, D. Pension schemes as options on pension fund assets: Implications for pension fund management. Insur. Math. Econ. 1998, 23, 263-286.

4. Chapman, R.J.; Gordon, T.J.; Speed, C.A. Pensions, funding, and risk. British Actuarial Journal 2001, 7(4), 605-663.

5. Campbell, J.Y.; Viceira, L.M. Strategic Asset Allocation: Portfolio Choice for Long-Term Investors; Clarendon Press: Oxford, UK, 2002

6. Cohen, J. How Many People Can the Earth Support? W.W. Norton \& Company: New York, NY, USA, 1995.

7. Gollier, C. Intergenerational risk-sharing and risk-taking of a pension fund. J. Public Econ. 2008, 92, 1463-1485.

8. Ponds, E.H.M. Pension funds and value-based generational accounting. JPEF 2003, 2(3), 295-325.

9. Møller, T.; Steffensen, M. Market-Valuation Methods in Life and Pension Insurance; Cambridge University Press: Cambridge, UK, 2007.

10. Ledermann, S. Nouvelles tables-types de mortalité; PUF/Ined: Paris, France, 1969.

11. Marini, P. L'épargne retraite en France trois ans après la loi Fillon (21 août 2003) : Quel complément de retraite par répartition? Rapport d'information fait au nom de la Commission des finances, du contrôle budgétaire et des comptes économiques de la Nation, Septembre 2006.

(c) 2013 by the author; licensee MDPI, Basel, Switzerland. This article is an open access article distributed under the terms and conditions of the Creative Commons Attribution license (http://creativecommons.org/licenses/by/3.0/). 